\title{
Static avalanches and Giant stress fluctuations in Silos
}

\author{
Philippe Claudin, Jean-Philippe Bouchaud \\ Service de Physique de l'Etat Condensé, CEA-Saclay, Orme des Merisiers, 91191 Gif s/ Yvette \\ CEDEX, France
}

(June 26, 2018)

\begin{abstract}
We propose a simple model for arch formation in silos. We show that small pertubations (such as the thermal expansion of the beads) may lead to giant stress fluctuations on the bottom plate of the silo. The relative amplitude $\Delta$ of these fluctuations are found to be power-law distributed, as $\Delta^{-\tau}, \tau \simeq$ 1.0. These fluctuations are related to large scale 'static avalanches', which correspond to long-range redistributions of stress paths within the silo.
\end{abstract}

Typeset using REVTEX 
Although granular matter is very familiar, it does display extremely interesting and unexpected features. For example, the stress distribution below a heap of sand shows a counter-intuitive minimum right below the apex of the pile [1], reflecting the presence of 'arches' (or line of forces) within the pile [2 4]. Another very common geometry is that of the silo. In this case, it is known since Janssen in 1895 that the weight $\mathcal{W}$ supported by the bottom plate of a tall silo is only a small fraction of the total weight of the grains contained in the silo - most of the weight is 'absorbed' by the side walls [5,66]. Very recent experiments actually reveal an even more striking effect: the 'apparent' weight $\mathcal{W}$ depends very sensitively on temperature [7]. More precisely, a variation of temperature of a few degrees induce rather erratic and abrupt changes of $\mathcal{W}$ of the order of a several $\%$, while relative change in the size of the grains due to thermal expansion is only $10^{-5}-10^{-4}$ ! Furthermore, these changes are of both signs and hysteretic: the apparent weight $\mathcal{W}$ is not a unique function of temperature. Another aspect of this phenomenon is the following: repeating the same experiment of filling a silo with precisely the same quantity of beads lead to an apparent weight $\mathcal{W}$ which fluctuates by more than $20 \%$ [7].

It has been long recognized that stress propagation within granular media is strongly inhomogeneous, with clear 'stress paths' appearing in birefringence experiments. This leads to large stress fluctuations which have been recently investigated experimentally [8], numerically and theoretically [8,9], through a simple 'scalar' model of stress propagation, where only the $z z$ (vertical) component of the stress tensor is considered. Although oversimplified, this model leads to predictions in qualitative and perhaps quantitative agreement with observations. From a theoretical point of view, it can be shown [10 that the full tensorial stress propagation model can indeed reduce, in some particular limits, to the scalar case considered by Liu et al. [8,9].

The basic idea to explain the 'giant' stress fluctuations induced by temperature changes (or any other small perturbation) is that the network of 'stress paths' is extremely sensitive to small perturbations. A stress path reaching a side wall (which can be seen as a partly absorbing boundary for the stress) might change its direction and rather reach the bottom 
plate, leading to a sudden upward 'jump' in $\mathcal{W}$. Conversely, the apparent weight can jump down as a path reaching the bottom plate is transformed into one colliding with the side walls.

The model we use to describe more quantitatively this effect is an extension of the one considered in [8,9] to allow for the formation of arches. For a two dimensional packing, each 'grain' is labelled by two integer $(i, n)$ giving its horizontal and vertical position. [Extension of the model to three dimensions is left for future work]. Each grain supports the weight of its two upstairs neighbours, and shares its own load randomly between its two downstairs neighbours. The corresponding scalar equation for weight propagation is thus:

$$
\begin{aligned}
W(i, n) & =q_{+}(i-1, n-1) W(i-1, n-1) \\
& +q_{-}(i+1, n-1) W(i+1, n-1)+w
\end{aligned}
$$

where $w$ is the weight of the grains, assumed to be constant. $q_{ \pm}(i, n)$ is the fraction of the weight transmitted to the the grain $(i \pm 1, n+1)$, and is a random variable between zero and one, subject to the mass conservation constraint $q_{+}(i, n)+q_{-}(i, n)=1$. The walls are at $i= \pm L$, and are simply defined by the fact that $q \pm( \pm L, n) \equiv 0$, and $q_{\mp}( \pm L, n)$ randomly chosen between zero and one; a fraction $1-q_{\mp}( \pm L, n)$ of the mass is thus 'absorbed' by the wall.

As shown by Liu et al. [8], when $q_{+}$is uniformly distributed, the resulting asymptotic weight distribution is given by $P\left(x=\frac{2 W}{\langle W\rangle}\right)=x \exp -x$, where $\langle W\rangle$ is the average weight. When $q_{+}=0$ or 1 , the distribution becomes much broader, decaying only as a power-law $W^{-\frac{4}{3}}[9]$.

We want to include the possibility that when the local shear on a given grain is too strong, this grain can 'lift off' from one of its downstairs neighbours and therefore only transmit its load to the neighbour which is in the direction of the shear. Since we work with a scalar model where shear is a priori absent, we assume that the transmitted shear stress from $(i, n)$ to $(i \pm 1, n+1)$ is also proportional to $q_{ \pm}(i, n) W(i, n)$, and thus postulate that if

$$
q_{+}(i-1, n-1) W(i-1, n-1)
$$




$$
-q_{-}(i+1, n-1) W(i+1, n-1)>\mathcal{R}_{c} W(i, n)
$$

(where $\mathcal{R}_{c}$ is a certain threshold), then $q_{-}(i, n)=0=1-q_{+}(i, n)$, meaning that the link to the left of $(i, n)$ is removed. In a symmetric fashion, if

$$
\begin{aligned}
& q_{-}(i+1, n-1) W(i+1, n-1) \\
- & q_{+}(i-1, n-1) W(i-1, n-1)>\mathcal{R}_{c} W(i, n)
\end{aligned}
$$

then $q_{+}(i, n)$ is set to zero. Note that for $\mathcal{R}_{c}=1$, the model of Liu et al. is exactly recovered (all the links are present). Conversely, for $\mathcal{R}_{c}=0$, the $q_{ \pm}$can only take values 0 or 1 .

This rule is interesting because it potentially leads to 'static' avalanches in the following sense: if $\mathcal{R}_{c}$ is slightly decreased, a link somewhere is removed - say $q_{-}(i, n)$. Then it is quite probable that the threshold will also be exceeded on site $(i+1, n+1)$, thereby inducing $q_{-}(i+1, n+1)$ to vanish, and so on. In other words, very long arches can suddenly appear or disappear when $\mathcal{R}_{c}$ is changed (see Fig. 3 below).

Physically, $\mathcal{R}_{c}$ should grow with the density $\phi=n a^{d}$ of the packing ( $d$ is the dimension of space), where $n$ is the number of grains per unit volume and $a$ the size of the grains. The reason is that obviously, contacts are easier to removed in a loose packing. It is thus reasonnable to assume that $\mathcal{R}_{c}$ will grow with temperature, with

$$
\frac{\delta \mathcal{R}_{c}}{\mathcal{R}_{c}} \simeq \frac{\delta a}{a}
$$

$\mathcal{R}_{c}$ presumably also depends on the friction coefficient between the grains, and is expected to be reach $\mathcal{R}_{c}=1$ in the limit of zero friction.

The total weight on the bottom plate of the silo is obviously defined as:

$$
\mathcal{W}=\sum_{i=-L}^{L} W(i, H)
$$

where $H$ is the height of the silo. Fig 1 shows $\mathcal{W}$ as a function of the threshold $\mathcal{R}_{c}$ for a given sample (i.e. a given set of $q_{ \pm}$uniformly chosen between zero and one [11]) and averaged over many samples (thick line), for a silo of size $D=2 L+1=61, H=610$. $\mathcal{W}$ is a constant for small $\mathcal{R}_{c}$, and then rapidly grows with $\mathcal{R}_{c}$, but reveals enormous fluctuations around the 
average trend. More precisely, we find that for a small change $\delta \mathcal{R}_{c}$ of the threshold, then with probability $\rho \delta \mathcal{R}_{c} \ll 1$ there is a 'static avalanche' in the sense that $\mathcal{W}$ changes by a finite amount, which is independent of $\delta \mathcal{R}_{c}$, and with probability $1-\rho \delta \mathcal{R}_{c}, \mathcal{W}$ does not vary at all. This picture is very similar to the 'shocks' in Burgers' turbulence [12]. Thus the full statistics of the $\mathcal{W}\left(\mathcal{R}_{c}\right)$ curve can be decomposed into two aspects: the density of avalanches, and the amplitude of these avalanches.

- The density $\rho\left(\mathcal{R}_{c}\right)$ of 'avalanches' or 'shocks' was obtained by plotting the probability that no shocks occur in an interval of length $\delta \mathcal{R}_{c}$, which is very well approximated by $\exp -\left[\rho \delta \mathcal{R}_{c}\right]$ (independent shocks). For $q$ uniformly distributed between zero and one, we found that $\rho\left(\mathcal{R}_{c}\right)$ is of the form $H L f\left(\mathcal{R}_{c}\right)$, where $f$ is a function of order 1 with increases rather rapidly with $\mathcal{R}_{c}$. Typically, for $H=200$ grain size and an aspect ratio of 1 , the density of shocks is $\sim 10000$.

- The relative value of the jump of $\mathcal{W}$ when an 'avalanche' occurs, will be denoted as $\Delta$. Interestingly, for an aspect ratio of 1 , but independently of $\mathcal{R}_{c}$ (and thus of the density $\rho$ ), $\Delta$ is distributed according to a power-law $\mathcal{P}(\Delta) \propto|\Delta|^{-\tau}$, with $\tau=1.02 \pm 0.03$ (see Fig. 2). On the largest sample $(H=D=201)$, this power law is observed on nearly five decades, cut-off for $\Delta$ smaller than a certain $\Delta^{*}$ (which decreases with $H$ ), and cut-off above $\Delta=1$, which corresponds to relative change of the weight of $100 \%$.

From a practical point of view, it means that for $H=D=201$ (in grain size units), a small change of $\delta \mathcal{R}_{c}$ as low as $10^{-5}$ will, with probability $\rho \delta \mathcal{R}_{c} \simeq 10^{-1}$ induce a relative change $\Delta$ of the apparent weight $\Delta$, distributed as $\Delta^{-\tau}$ up to $\Delta \simeq 1$ ! Since $\tau$ is small (close to 1 ), huge relative changes of the weight between say $10^{-2}-10^{-1}$ have a probability given by $\frac{1}{\log _{10} \Delta^{*}}$, which is of the order of 0.2 . Hence, small perturbations typically induce large responses.

Fig 3 shows the 'stress paths' (i.e. grains on which the load a certain arbitrary value) for two very close values thresholds such that $\mathcal{W}$ has changed dramatically. A small perturbation indeed induces a noticable rearrangment of the stress paths (which could perhaps be directly detected by acoustic emission [13]). This phenomenology is actually very close to the one 
of quantum conductance fluctuations in strongly disordered samples, where small changes of the chemical potential or the magnetic field lead to a complete change in the optimally conducting paths [14]. The log of the conductance then also shows large variations. Another similar situation is that of 'directed polymers' in random environments, where small changes of the local (disordered) potential, or a small external force, can lead to a large-scale change in the ground state conformation [15 18]. In the same family of problems, one should actually cite spin-glasses, which have also been argued to be 'chaotic', i.e. very sensitive to temperature or disorder changes [19,20].

An interesting consequence of our model is that in the region where small perturbations lead to substantial effects, there is a large fraction of links which take a value $q_{ \pm}=0,1$. This is turn, according to the arguments of [9], should lead to a power-law distribution $W^{-\frac{4}{3}}$ for the local stress on the bottom plate. Fig 4 shows that the stress distribution for $\mathcal{R}_{c}=0.9$ can be fitted to $W^{-1.18}$. Note however that the arguments of [9] assume no correlations between the $q_{ \pm}=0,1$, which is not the case here since broken links appear precisely along arches. For $\mathcal{R}_{c}=1$, the distribution has exponential tails [9]. We thus expect that these giant fluctuations tend to disappear when the density of the packing is increased, or when the friction between the grains decreases.

When the aspect ratio $\frac{H}{D}$ increases, the situation changes in the following way. When a static avalanche occurs 'far' from the bottom plate, the resulting change of weight is screened out by the presence of the (partly) absorbing walls. A simple argument allows one to understand that the avalanche size distribution $\mathcal{P}(\Delta)$ is now cut-off beyond a certain $\Delta_{\max }$ which decreases as the aspect ratio increases. This is precisely what we observe numerically. Furthermore, one observes a progressive 'localisation' of the stress on a unique path, which, for large aspect ratios and small $\mathcal{R}_{c}$, carries a finite fraction of $\mathcal{W}$.

Finally, let us discuss the above phenomenon from a slightly different point of view. Instead of fixing the disorder (i.e. the $q_{ \pm}$) and changing the threshold $\mathcal{R}_{c}$, one can fix $\mathcal{R}_{c}$ and change the disorder. This would correspond to repeating the same experiment of filling a silo, thereby building different local arrangments of the grains. As expected by a kind 
of ergodicity argument, the resulting distribution of apparent rescaled weight $\frac{\mathcal{W}}{\langle\mathcal{W}\rangle}$ is quite broad. As soon as the aspect ratio exceeds 5, this distribution is very close to a gaussian and independent of $H$, with a relative root mean square of $\sim 20-30 \%$. This compares rather well with the experiments. In the same spirit, hysteretic effects can be accounted for by the fact that when a contact is lost and then reformed when the temperature comes back to its initial value, the new values of $q_{ \pm}$have no reason to be the same as before, since the local environment has slightly changed.

We have also investigated this model in the heap (sandpile) geometry. When $\mathcal{R}_{c}=1$, the stress distribution at the bottom of the pile is maximum just below the apex of the pile. However, when $\mathcal{R}_{c}$ is less than a certain critical value, arches spanning the size of the system suddenly proliferate, and a dip at the center of the pile is created, as observed experimentally. The importance of long arches to understand the 'dip' was stressed in [2, 幽. The present model could be a microscopic foundation of this idea.

As a conclusion, we have shown that a motivated extension of the model proposed by Liu et al. to account for arch formation can explain the rather spectacular giant stress fluctuations induced by small temperature changes. The basic idea is that of long-range modification of stress paths induced by a small perturbation, which can be seen as 'static avalanches'. Actually, our model is rather close to the large class of 'SOC' (Self-Organized Critical) models [21,22], initially proposed to describe true 'dynamical' avalanches in granular media. The irony would be that these scale invariant avalanches should rather be looked for in static properties !

Acknowlegments. We want to thank M.E. Cates, E. Clément, J. Duran, J. Rajchenbach and J. Wittmer for many fruitful discussions.

\section{Figure Captions}

Fig 1: Evolution of the apparent weight $\mathcal{W}$ as a function of the threshold for a silo of size $D=61$, and $H=601$. The thick line is an average over 1000 samples, while the thin 
line is a particular realisation of the disorder.

Fig 2: Distribution of shock amplitudes $\Delta$, in $\log$-log coordinates, for $\mathcal{R}_{c}=0.6$ and $D=L=201$ (thick line), and $D=L=21$ (dotted line). For the larger sample, the distribution is very nearly given by $\Delta^{-1}$ between $10^{-6}$ and $10^{-1}$.

Fig 3: Chaoticity of stress paths. We show the large stress paths for two very close values of $\mathcal{R}_{c}$, separated by a shock, and $H=L=201$. For the second value of $\mathcal{R}_{c}$, the paths have been translated by $x \rightarrow x+2$ for clarity. As one can see, some large scale features of the stress network have changed; the total weight carried by the bottom plate has changed $\mathcal{W}=0.735$ to $\mathcal{W}=0.845 !$

Fig 4: Distribution of the local stress $W_{i}$ on the bottom plate, for $\mathcal{R}_{c}=0.9$ and a sample of size $H=201, D=201$. The best fit gives an exponent $-1.18 \pm 0.04$ for the exponent. This value is not far from the value $-\frac{4}{3}$ expected from the analysis of Coppersmith et al. [9], valid when $q_{ \pm}$are independent and can only take the values 0 or 1 . 


\section{REFERENCES}

[1] Smid, J. and Novosad, J. in Proc. of 1981 Powtech Conference, Ind. Chem. Eng. Symp. 63, D3V 1-12 (1981).

[2] Edwards, S. F. and Oakeshott, R. B. Physica D 38, 88-93 (1989); see also Edwards, S. F. and Mounfield, C. C., Physica A 226, 1, (1996)

[3] Bouchaud, J.-P., Cates, M. E. and Claudin, P., J.Physique I 5639 (1995).

[4] Wittmer, J., Claudin, P., Cates, M. E. and Bouchaud, J.-P., Nature 382336 (1996), Wittmer, J. Cates, M. E. and Claudin, P., submitted to J. Physique.

[5] Brown, R. L. and Richard, J. C. Principles of Powder Mechanics (Pergamon, New York 1966).

[6] Nedderman, R. M. Statics and Kinematics of Granular Materials (Cambridge University Press, 1992); Sokolovskii, V. V. Statics of Granular Materials (Pergamon, Oxford 1965).

[7] E. Clément, J. Duran, J. Rajchenbach, private communication.

[8] C. H. Liu et al., Science 269 513-515 (1995).

[9] S. Coppersmith, C. H. Liu, S. Majumdar, O. Narayan, T. Witten. Phys. Rev. E 53 (1996) 4673.

[10] P. Claudin, J. Wittmer, J.P. Bouchaud, M. E. Cates, in preparation.

[11] More generally, $q$ is chosen uniformly in the interval $\left[\frac{1-\epsilon}{2}, \frac{1+\epsilon}{2}\right]$. ). As one could expect, all the results obtained below qualitatively hold independently of $\epsilon$. For example, the existence of a power-law regime, and the value of the exponent $\tau$ are independent on the strength of the disorder $\epsilon$.

[12] J. M. Burgers, 'The Non-Linear Diffusion Equation', D. Reidel Pub. Co. (1974), S. Kida, J. Fluid. Mech. 93 (1979) 337. 
[13] This noise is similar to the Barhausen noise in magnets, which reflects the sudden change of the domain wall positions, much like the change of stress paths proposed here.

[14] For a review, see: 'Mesoscopic Phenomena in Solids', B. Altshuler, P.A. Lee and R.A. Webb Edts, (Elsevier Science Publisher, 1991). See also: F. Ladieu, J.P. Bouchaud, J. Phys. I France 3 (1993) 2311.

[15] M. Mézard, J. Phys. France 511831 (1990)

[16] G. Parisi, J. Phys. France 511595 (1990)

[17] T. Hwa, D. S. Fisher, Phys. Rev. B 493136 (1994).

[18] T. Halpin-Healey and Y.C. Zhang; Phys. Rep. 254 (1995) 217.

[19] A. J. Bray, M. A. Moore, Phys. Rev. Lett. 58 (1987) 57.

[20] D. S. Fisher and D. A. Huse, Phys. Rev. B 38, 373 (1988) and ibid. 38, 386 (1988).

[21] P. Bak, C. Tang, K. Wiesenfeld, Phys. Rev. Lett. 59, 381 (1987), Phys. Rev. A 38, 364 (1988).

[22] For a review, see G. Grinstein, in 'Scale Invariance, Interfaces and Non-equilibrium dynamics', NATO-ASI, Kluwer (1995). 
silo $61 \times 610$

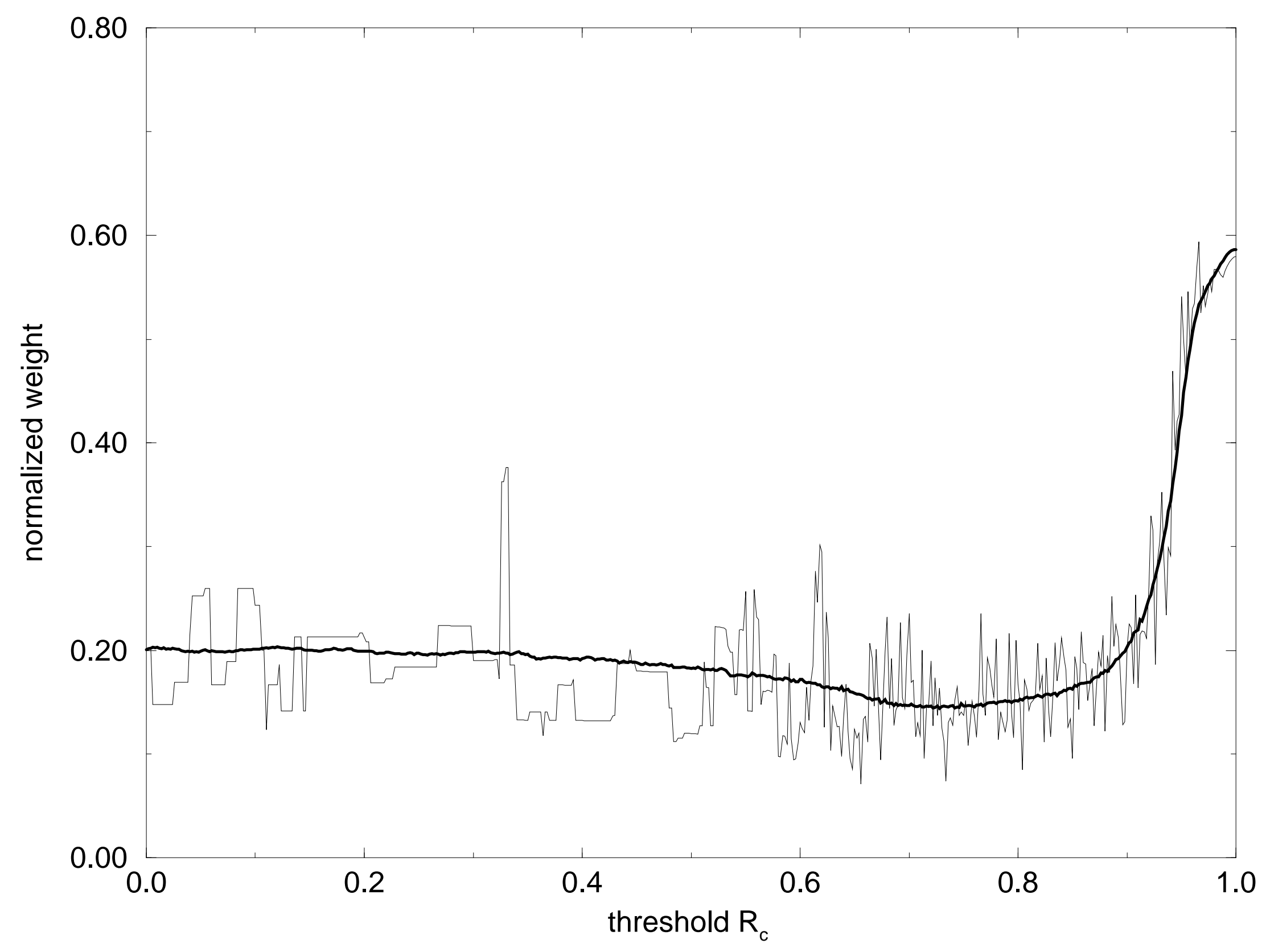


Shock Amplitude Distribution

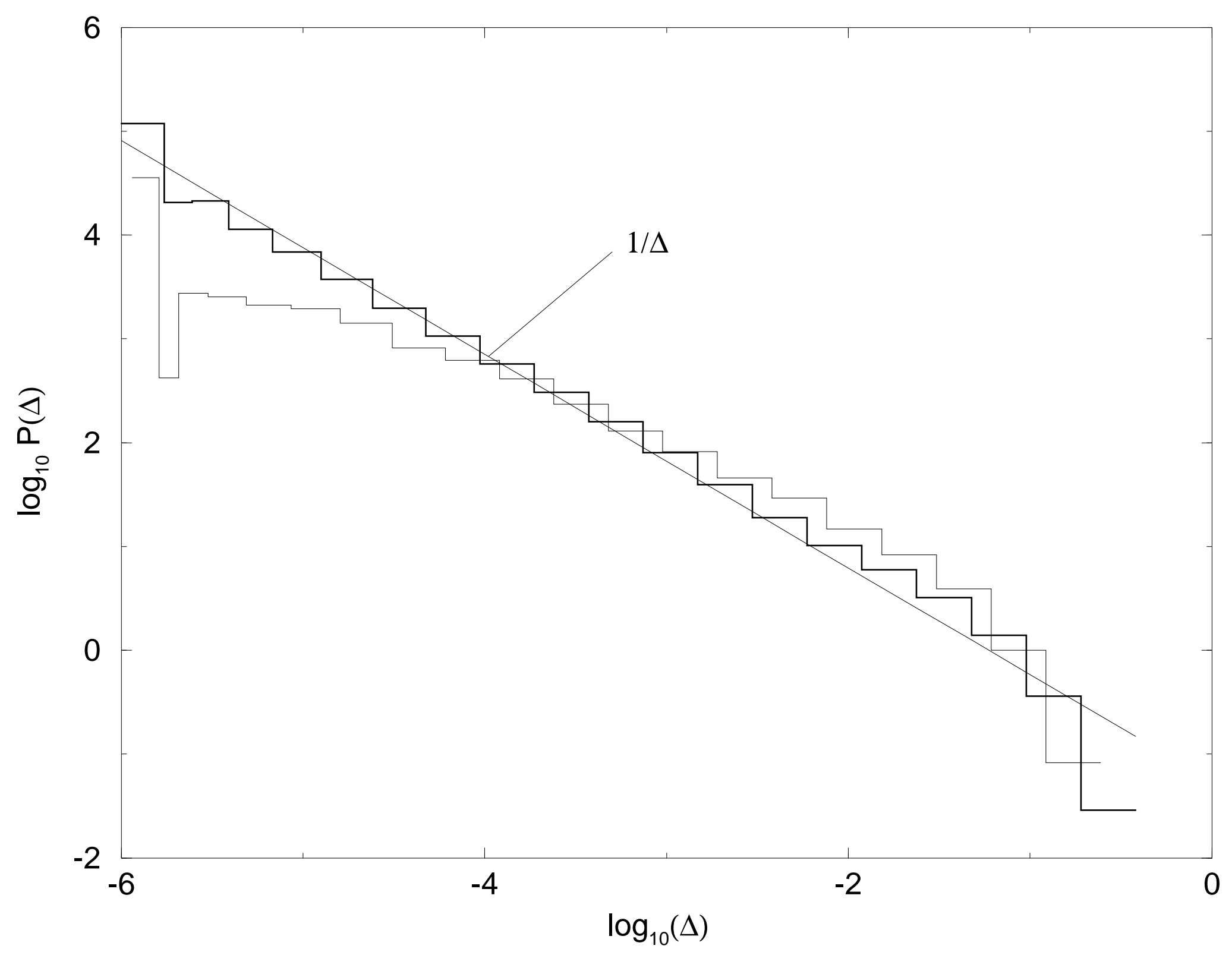




\section{Stress Paths $\left(w>5 w_{a v}\right)$}

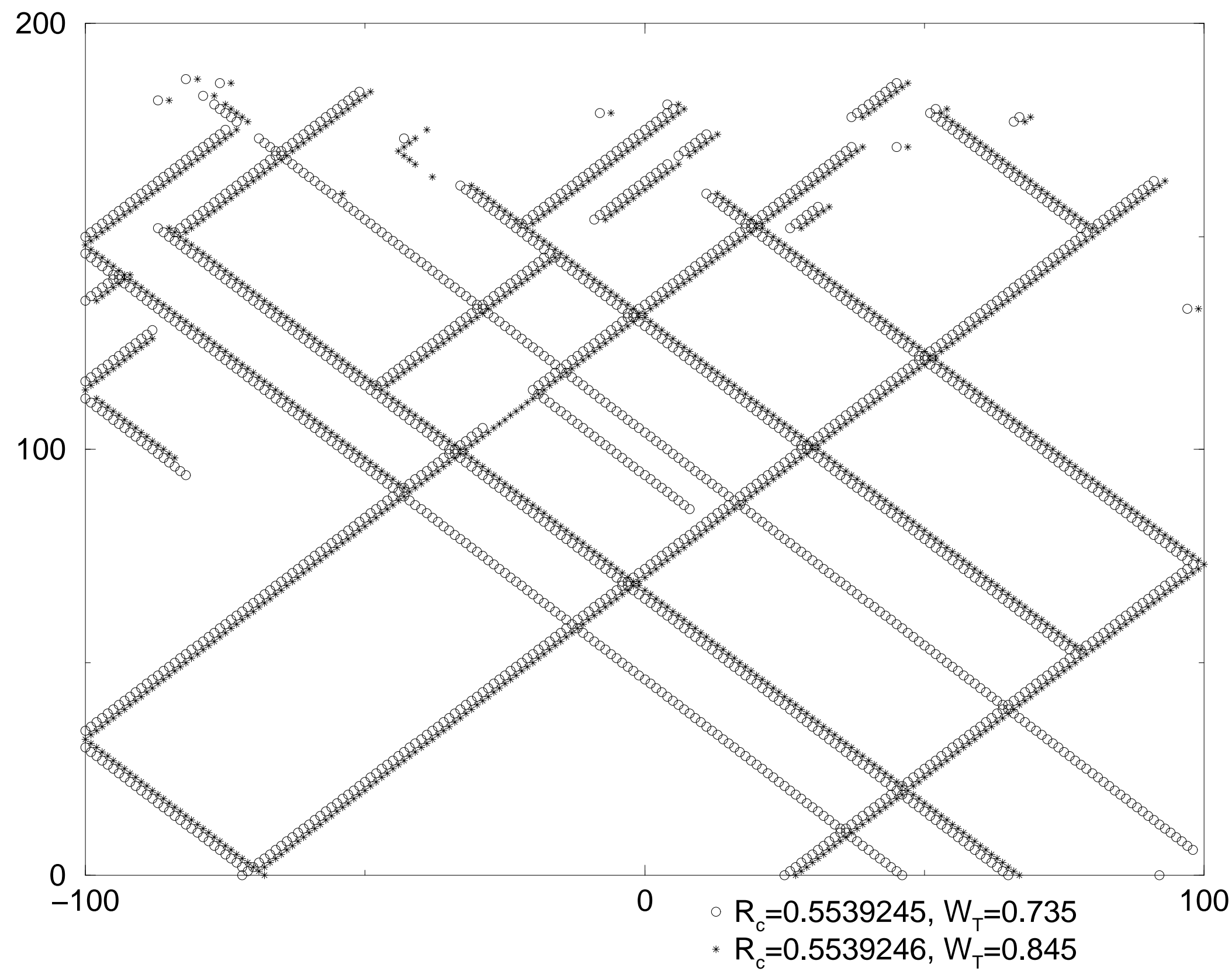




\section{Weight Distribution}

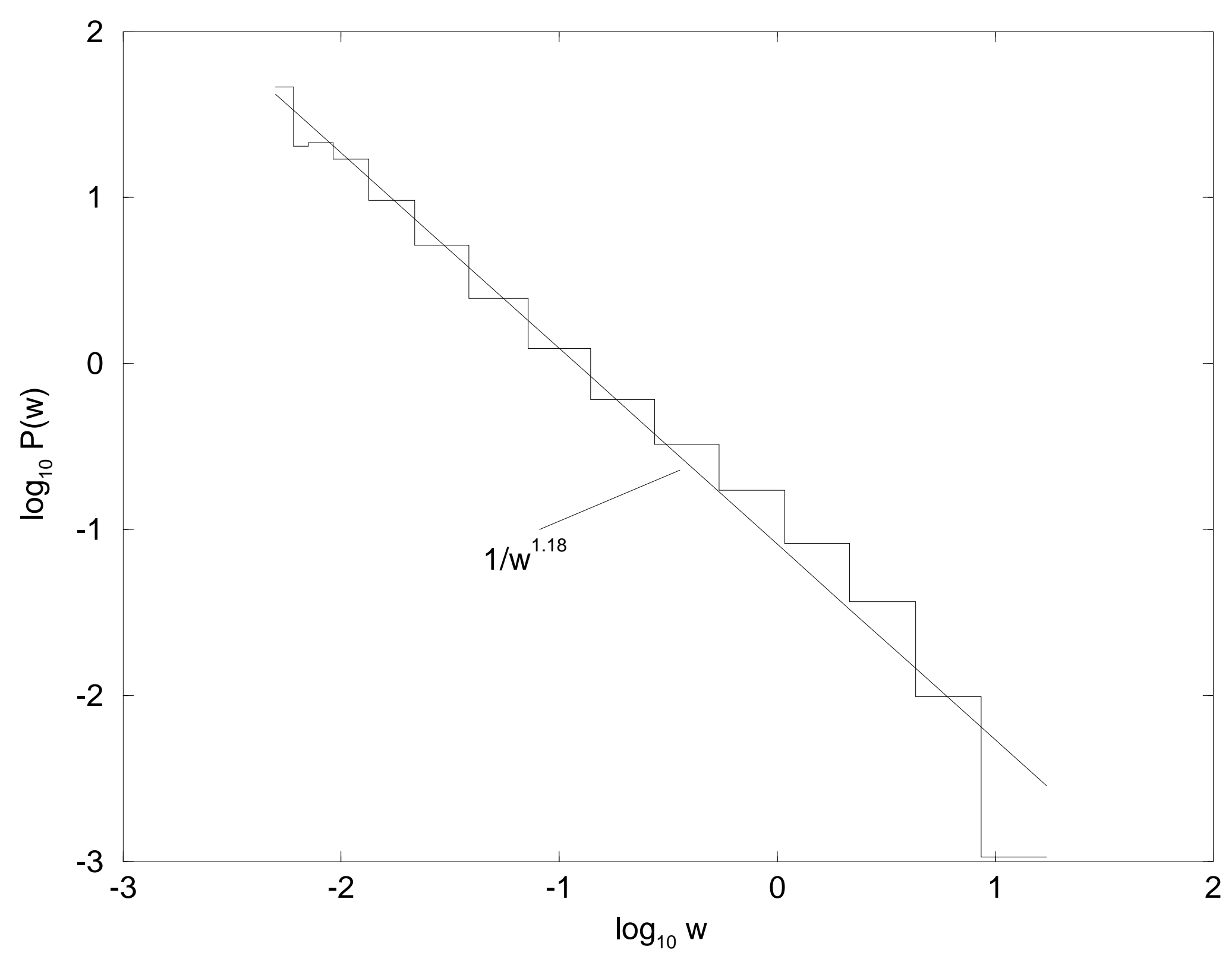

